\title{
An Uptated View on the Approach to Tricuspid Regurgitation
}

\author{
Clara Weksler \\ Instituto Nacional de Cardiologia, Rio de Janeiro, RJ - Brazil. \\ Editorial referring to the article: Early Outcomes of Modified De Vega Annuloplasty for Functional Tricuspid Regurgitation at a Brazilian Hospital
}

The incidence of tricuspid regurgitation (TR) associated with left valvular disease is significant ranging from $8 \%$ to $35 \%$ of cases. ${ }^{1,2}$ This is most common in conjunction with mitral valve disease but association with aortic valve pathology is not uncommon and frequently related to rheumatic valve disease and much rarer in association with degenerative mitral valve disease. In most cases, the tricuspid regurgitation is so called "functional", in other words, secondary to dilatation of the annulus as a consequence of right ventricular (RV) dilatation and pulmonary hypertension. The functional tricuspid regurgitation is a progressive disease that does not always resolve with the correction of the left-side lesion. The recommendations that existed were generally conservative, late in timing, and reflective in large measure of the historically poor outcomes with Tricuspid Valve (TV) surgery in the context of delayed referral, advanced heart failure symptoms, and RV dysfunction

It has become evident that in a significant number of cases, secondary TR does not regressed after appropriate correction of the left-side disease leading to a more aggressive conduct. ${ }^{3,4}$

Current ESC guidelines 2017 suggest that surgery should be considered in patients with mild or moderate secondary TR with annulus $>40 \mathrm{~mm}$ or $21 \mathrm{~mm} / \mathrm{m}^{2}$ undergoing left-side valve surgery (Class IIa indication, level of evidence C). ${ }^{5}$

The American Heart Association /American College of Cardiology (AHA/ACC) guidelines 2014 also recommended in patients with pulmonary hypertension (Class IIa indication, level of evidence C). ${ }^{6}$

\section{Keywords}

Tricuspid repair; Mitral and aortic disease; Pulmonary hypertension

Mailing Address: Clara Weksler

Rua das Laranjeiras 374. Postal Code: 22240-006, Rio de Janeiro, RJ - Brazil. E-mail: weksler.clara@gmail.com

DOI: https://doi.org/10.36660/ijcs.20200325
In those with isolate severe TR, surgery is recommended in the presence of symptoms or progressive right ventricular dilatation or dysfunction. ${ }^{7}$

Tricuspid intervention at the time of the surgery to the left side valve usually helps to improve the functional capacity without significant increase in perioperative mortality and morbidity

Before left-side valve surgery, careful assessment of the severity of TR and careful measurement of the tricuspid annulus is mandatory.

The patients may remain asymptomatic even with TR of moderate or severe degree. When symptoms appear, patients may complain asthenia, fatigue or decreased exercise tolerance as a result of lower cardiac output. The signs of elevated right atrial pressure, such as peripheral oedema and abdominal fullness, congestive hepatomegaly and ascites will be present in the evolution of disease. Atrial fibrillation as a result of right atrial enlargement is common

Evaluation of patients with TR requires the integration of the information from different cardiac imaging techniques. The transthoracic echocardiography (TTE) and cardiovascular magnetic resonance(CMR) are the most used (Table 1). ${ }^{8}$

Transthoracic echocardiography is the technique of choice to evaluate the aetiology of the TR, quantify its severity and determine the annular diameters. The normal annulus diameter in adults is $28 \pm 5 \mathrm{~mm}$ and a significant dilation is defined by a diastolic diameter $>40 \mathrm{~mm}$ or $21 \mathrm{~mm} / \mathrm{mm} .{ }^{2,8}$

The selection of a valve repair versus replacement is largely driven by anatomic factors, including the extent of leaflet damage and degree of anular dilatation. When feasible, valve repair may be preferred due to the risks of prosthetic valve thrombosis, bioprosthetic valve degeneration and long term anticoagulation, Repair is generally favoured in patients undergoing left-sided surgery as these techniques can be accomplished quickly to minimise bypass time.It is estimated that $73 \%$ of TV operations are repairs, with $88 \%$ performed 
Table 1 - Echocardiographic (A) and cardiovascular magnetic resonance (B) reference values of RV and right atrial size and function in healthy adults

\section{Abnormal}

(A) Echocardiography

\begin{tabular}{|c|c|}
\hline \multicolumn{2}{|l|}{ RV diameter (mm) } \\
\hline Base $^{*}$ & $>41$ \\
\hline Midventricular Level ${ }^{*}$ & $>35$ \\
\hline Length* & $>83$ \\
\hline RV wall thickness (subcostal view) (mm) & $>5$ \\
\hline RA end-systolic area (mm2) & $>18$ \\
\hline RA volume (mL/m2) & $>30$ \\
\hline \multicolumn{2}{|l|}{ Systolic function } \\
\hline TAPSE (mm) & $>17$ \\
\hline Pulsed Doppler peak 5' (m/s) & $<9.5$ \\
\hline RV fractional area change $(\%)$ & $<35$ \\
\hline RV 3D EF (\%) & $<45$ \\
\hline \multicolumn{2}{|l|}{ Diastolic Function } \\
\hline $\mathrm{E} / \mathrm{E}^{\prime}$ ratio & $>6$ \\
\hline Tissue Doppler MPI & $>0.54$ \\
\hline \multicolumn{2}{|l|}{ (B) Cardiovascular magnetic resonance } \\
\hline End-diastolic volume/BSA (mL/m2) & $>108$ \\
\hline End-systolic volume/BSA (mL/m2) & $>48$ \\
\hline $\mathrm{EF}(\%)$ & $<50$ \\
\hline Mass (g/m2) & $>46$ \\
\hline
\end{tabular}

at the time of left-sided valve surgery. ${ }^{9}$ The most common surgical techniques are reviewed in figure 1

It is generally believed that the long-term results of tricuspid annuloplasty are more favorable than those obtained after valve replacement, whether by mechanical or bioprosthetic implants. The survival after replacement has been reported as low as 35\% and up to $75 \%$ after 10 years These numbers are lower than those that we observed after tricuspid annuloplasty

The surgical technique described by Ferraz et al entitled Early Outcomes of Modified De Vega Annuloplasty for Functional Tricuspid Regurgitation at a Brazilian Hospital a modified De Vega technique ${ }^{10}$ by interposition of Teflon felt pledgets for each annular bite of the suture, produced excellent results.

Yan Topilsky and cols investigated the impact of TR in 271 patients with left ventricular systolic dysfunction (ejection fraction $31 \pm 10 \%$ ), functional tricuspid regurgitation and an effective regurgitation orifice area of $0,26 \pm 0,3 \mathrm{~cm}^{2}$ on clinical outcome. Presentation with right heart failure was strongly related to the degree of TR. An effective regurgitant orifice area $\geq 0,4 \mathrm{~cm}^{2}$ was associated with increased mortality and increased cardiac events including mortality, new atrial fibrillation or heart failure (figure 2$)^{11}$ 

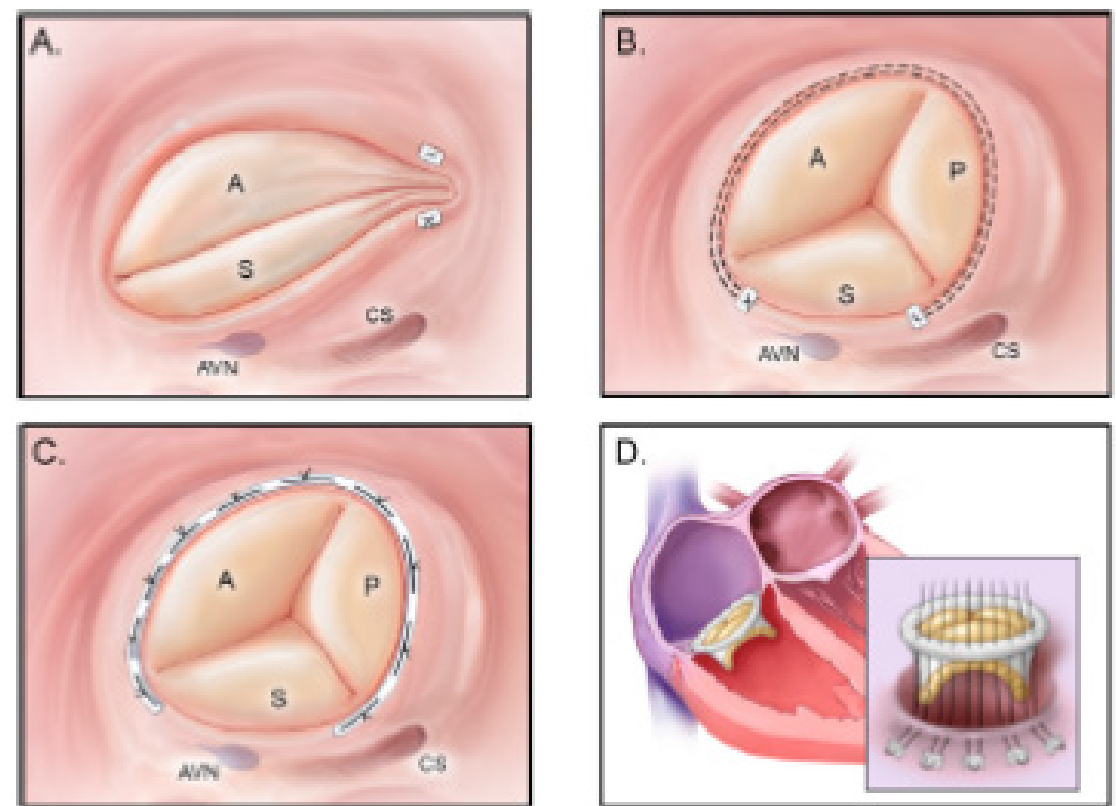

Figure 1 - The most common tricuspid valve operations include the Kay bicuspidisation (A), DeVega suture annuloplasty (B), prosthetic annuloplasty band (C) and tricuspid valve replacement (D).AVN, atrioventricular node; CS, coronary sinus; A, anterior leaflet; P, posterior leaflet; S, septal leaflet

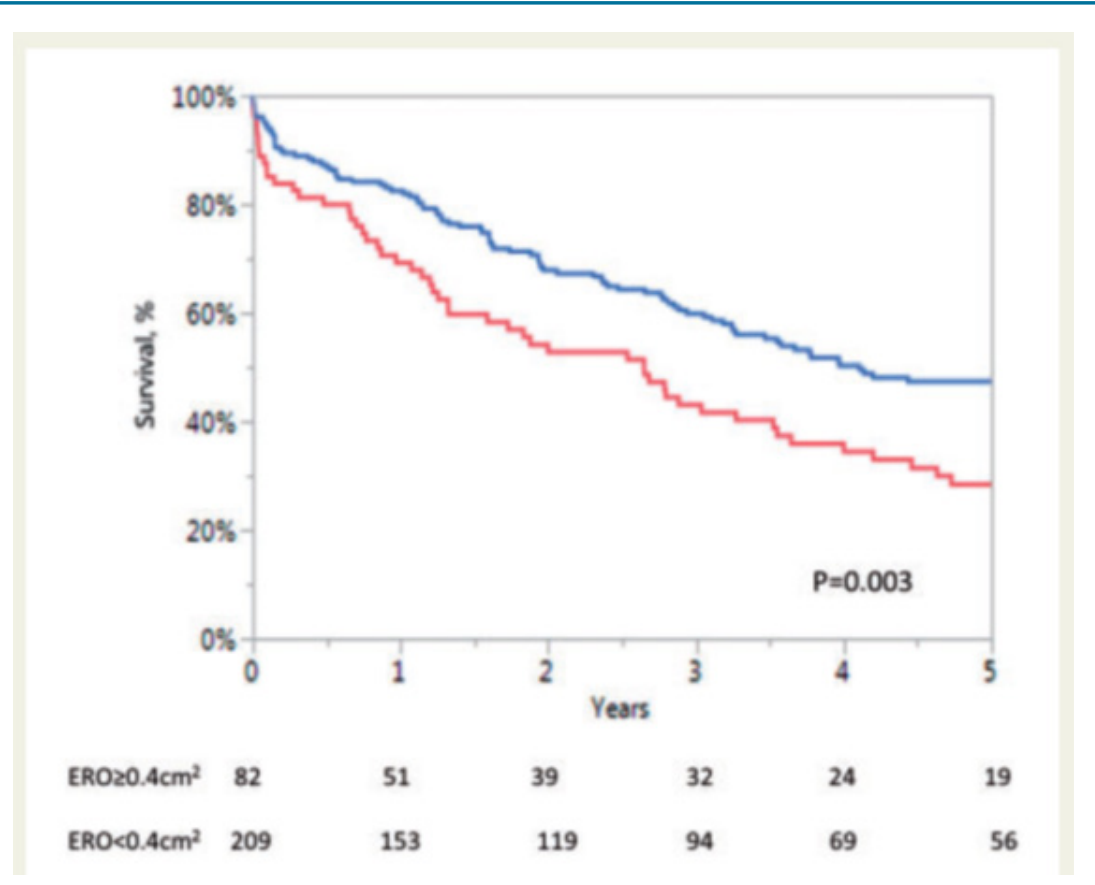

(EROA $<0.4 \mathrm{~cm}^{2}$, blue line; blue line; $\geq 0.4 \mathrm{~cm}^{2}$, red line)

Figure 2 - Overall survival under medical management in patients with tricuspid regurgitation associated with systolic dysfunction comparing patients with severe tricuspid regurgitation (effective regurgitant orifice $\geq 0.4 \mathrm{~cm}^{2}$ ) to lesser degree of tricuspid regurgitation (effective regurgitant orifice $<0.4 \mathrm{~cm}^{2}$ blue line, $\geq 0.4 \mathrm{~cm}^{2}$ red line). Note that there is decrease in survival with effective regurgitant orifice $\geq 0.4 \mathrm{~cm}^{2}$ 
Enthusiasm for the application of transcatheter tricuspid valve therapies to the treatment of severe TR has logically followed in the wake of the successes achieved with aortic and mitral valve interventions. The principles learned regarding clinical evaluation, multimodality imaging algorithms, risk assessment, multidisciplinary team consensus treatment recommendations, and shared decision-making are directly transferable. Although it is attractive to consider the use of less invasive, nonsurgical interventions earlier in the natural history of TR to prevent its long-term deleterious consequences, the effectiveness, safety, and durability of catheter-based treatments must first be established and then compared against current medical and surgical standards ${ }^{9}$ (figure 3 )

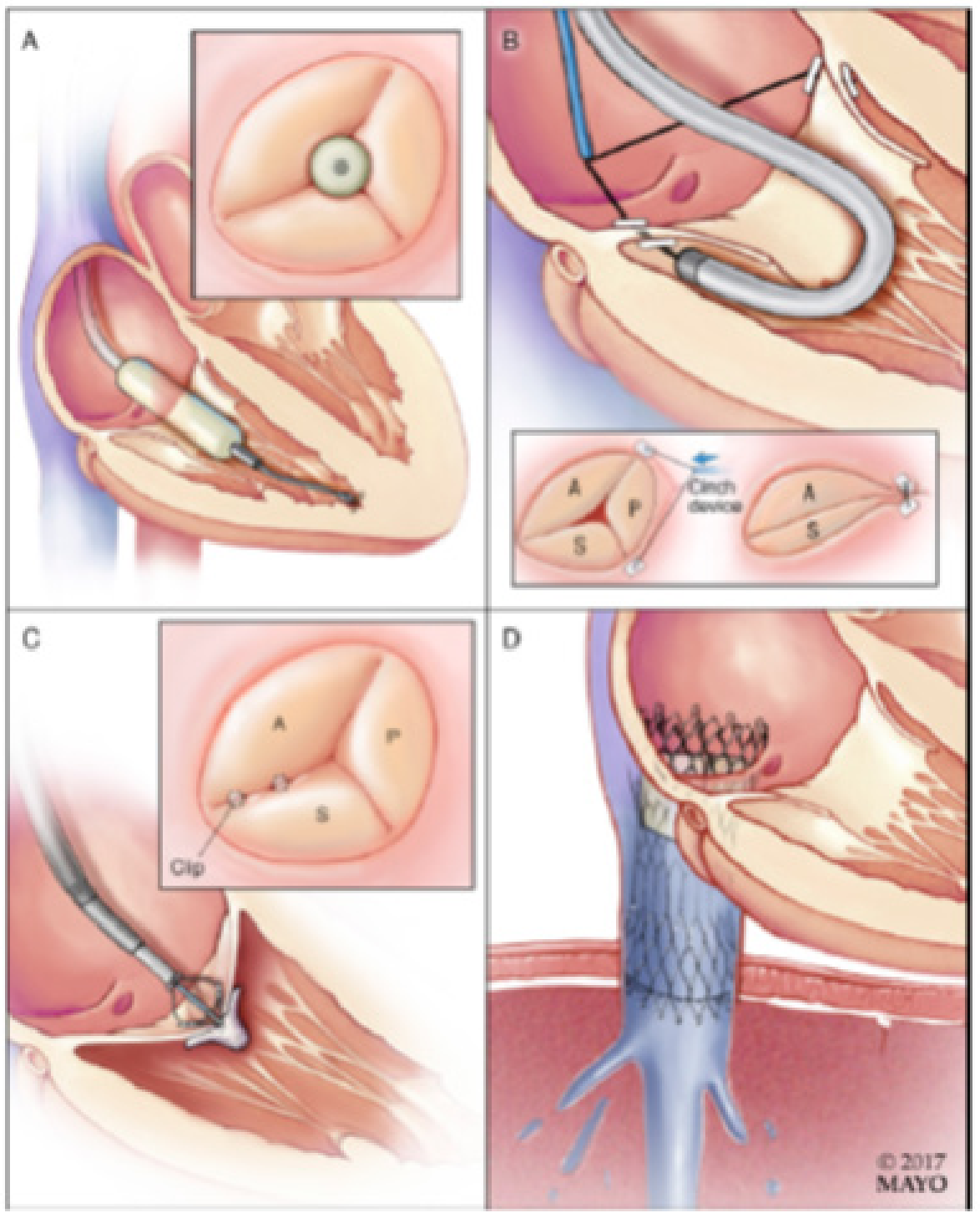

Figure 3 - Percutaneous devices in development for the treatment of tricuspid regurgitation. Panel (A) is the FORMA device, a tricuspid spacer which occupies the regurgitant orifice and provide a surface against which coaptation can occur. Panel (B) demonstrates the TriAlign, which percutaneously reproduces a surgical Kay bicuspidisation. Panel (C) shows the MitraClip being used in the tricuspid position. Panel (D) demonstrates a stented caval valve implanted in the inferior vena cava. 


\section{References}

1. Jacovella G, Marsocci G, Vajola FS, Masini V. Indications for surgical correction of tricuspid defects. Cardiol Prat, 1971;22(4):235-41.

2. King RM, Schaff HV, Danielson GK, Gersh BJ, Orszulak TA, Piehler JM, et al. Surgery for tricuspid regurgitation late after mitral replacement. Circulation. 1984;70(3Pt2):1193-7

3. Colombo T, Russo C, Ciliberto GR, Lanfranconi M, Bruschi G, Agati S, et al. Tricuspid regurgitation secondary to mitral valve disease: tricuspid annulus function as guide to tricuspid valve repair. Cardiovasc Surg 2001;9(4):369-77.

4. Dreyfus GD, Corbi PJ, Chan KM, Bahrami T. Secondary tricuspid regurgitation or dilatation: which should be the criteria for surgical repair? Ann Thorac Surg. 2005;79(1):127-32 .

5. 2017 ESC/EACTS Guidelines for the management of valvular heart disease. The Task Force for the Management of Valvular Heart Disease of the European Society of Cardiology (ESC) and the European Association for Cardio-Thoracic Surgery (EACTS) European Heart Journal (2017) 00, 1-53.

6. Nishimura RA, Otto C, Bonow R, Carabello BA, Erwin 3rd JP, Guyton RA, et al. 2014 AHA/ACC Valvular Heart Disease Guideline. J Am Coll Cardiol.2014;63(22):2438-88.
7. Irwin RB, Luckie M, Khattar RS. Tricuspid regurgitation:contemporary management of a neglected valvular lesion. Postgrad Med J. 2010;86(1021):648-55

8. Lang RM, Badano LP, Mor-Avi V, Afilalo J, Armstrong A, Ernande L, et al. Recommendations for cardiac chamber quantification by echocardiography in adults: an update from the american society of echocardiography and the European association of cardiovascular imaging. J Am Soc Echocardiogr .2015;28:1-39. e 14

9. Vassileva CM, Shabosky J, Boley T, Markwell S, Hazebugg S. Tricuspid valve surgery: the past 10 years from the Nationwide Inpatient Sample (NIS) database. J Thorac Cardiovasc Surg. 2012;143(5):1043-9.

10. Ferraz DLM, Alves KMB, Santos LAB, Leandro GS, Cunha CBC, Tchaick RM et al. Early Outcomes of Modified De Vega Annuloplasty for Functional Tricuspid Regurgitation at a Brazilian Hospital. Int. J. Cardiovasc. Sci.2020;33(5):472-478.

11. Topilsky Y, Inojosa JM, Benfari G, Vaturi O, Maltais S, Michelena H, Mankad S, Enriquez -Sarano M. Clinical presentation and outcome of tricuspid regurgitation in patients with systolic dysfunction. Eur Heart J. 2018;39(39):3584-92. 